\title{
ACOUSTIC-GRAVITY WAVES IN A VISCOUS AND THERMALLY CONDUCTING ISOTHERMAL ATMOSPHERE
}

(Part I. For large Prandtl number)

\author{
H. Y. ALKAHBY
}

\author{
Department of Mathematics and Computer Science \\ Adelphi University \\ Garden City, NY 11530
}

(Recelved April 14, 1993 and in revised form May 24, 1993)

\begin{abstract}
In this paper we will investigate the effect of Newtonian cooling on the propagation of acoustic-gravity waves in a viscous and thermally conducting isothermal atmosphere for large Prandtl number and for an arbitrary values of Newtonian cooling coefficient. This problem leads to a singular perturbation problem which is solved by matching inner and outer approximations. It is shown that the viscosity creates an absorbing and reflecting layer. Below it the oscillatory process is adiabatic, for small Newtonian cooling coefficient, and above it the solution will decay to constant before it is influenced by the effect of the thermal conductivity. Newtonian cooling is a volume effect and influences mainly the lower adiabatic region, in which it causes attenuation in the amplitude of the wave. Finally it is shown that when Newtonian cooling coefficient goes to infinity it acts directly to eliminate the temperature perturbation associated with the wave and the attenuation factor in the amplitude of the wave. Accordingly the wavelength changes to the one consistent with the Newtonian sound speed. The reflection coefficient and the attenuation factor of the amplitude of the wave are derived for all values of Newtonian cooling coefficient.
\end{abstract}

KEY WORDS: Acoustic-Gravity Waves, Atmospheric Waves, Wave Propagation AMS SUBJECT CLASSIFICATION CODES. 76N, 76Q

\section{INTRODUCTION}

The propagation of acoustic-gravity waves in isothermal and non-isothermal atmospheres has been extensively studied in recent years. Much of the motivations of these studies comes from their relevance to phenomena in compressible ionized fluids, such as solar, stellar, earths atmospheres and to certain phenomena in ocean dynamics ( Alkahby and Yanowitch[1989,1991], Campos[1983a,b], Debnath and Basu[1984], Lyons and Yanowitch[1974], Lindzen[1968,1970], Parker[1979], Priest[1982], Roberts[1968], Soward[1986], Webb and Roberts[1980]).

The reflecting properties of a viscous and thermally conducting isothermal atmosphere for small Prandtl number were investigated in Lyons and Yanowitch[1974]. It was shown that there are three 
distinct regions connected by two different reflecting layers. The lower region is adiabatic and in it the effects of the viscosity and the thermal conductivity are negligible, while the effects of these quantities are large in the upper region. In the middle region the effects of the thermal conduction is large while that of the viscosity is still negligible. The lower two regions are connected by a semitransparent reflecting layer, created by the exponential increase of the thermal diffusivity with height, allowing part of the energy to propagate upward and the remaining part to be reflected downward. The upward propagating waves in the isothermal region will be reflected downward by a second reflecting layer created by the exponential increase of the kinematic viscosity with altitude, which acts like an absorbing and reflecting barrier. Below it the oscillatory process is isothermal and above it the motion will decay to a constant value. A similar problem is considered by Alkahby and Yanowitch [1991], in which the combined effects of the thermal conduction and a horizontal magnetic field are investigated. In these two problems the existence of two reflecting layers will influence the motion in the lower region and the final conclusions depend on the relative locations of the two reflecting layers.

Newtonian cooling, which refers to the heat exchange proportional to the temperature perturbation, is important enough to be considered in many acoustic gravity wave and magneto acoustic gravity wave problems (Alkahby and Yanowitch[1989], Cally[1984], Mihalas and Toomre[1981,1982], Lindzen[1968,1970], Zhugzhda[1979], Webb and Roberts[1980]). The motivation for investigating the effects of Newtonian cooling on the wave motions relate to the energy balance in the solar atmosphere. Thus to estimate the mechanical energy flux that some type of waves can transform from the convective zone into the chromosphere, we have to estimate both energy flux initially generated by the convective zone and the fraction of that flux dissipated in the photosphere by radiative damping.

In this paper we will investigate the effects of Newtonian cooling on the vertical propagating acoustic-gravity waves in a viscous and thermally conducting isothermal atmosphere for large Prandtl number and an arbitrary values of Newtonian cooling coefficient. This problem leads to a singular perturbation problem which is solved by matching inner and outer approximations for the solutions in an overlapping domain.

It is shown that viscosity creates an absorbing and reflecting layer. Below it the motion is adiabatic and for frequencies above the adiabatic cutoff frequency the solution can be represented by a linear combination of an upward and downward propagating wave. Above it the the solution will decay exponentially with the altitude to a constant value before it is influenced by the effects of the thermal conductivity. Thus the effects of the thermal conduction on the wave propagation will be negligible. We have concluded that as the viscosity tends to zero the reflecting layer recedes to infinity, but the ratio of the amplitudes of the reflected to the incident wave tends to a limit. The solution, however, does not approach a limit at fixed values of the vertical coordinate $\mathrm{z}$, for the shift of the reflecting layer causes a change in the relative phase between the two waves.

Newtonian cooling, on the other hand, is a volume effect and will influence mainly the adiabatic region. It is shown that if Newtonian cooling coefficient is large compared to the adiabatic cutoff 
frequency, the heat exchange due to the radiation will be intense enough to eliminate the magnitude of the temperature perturbation associated with the wave in a time small compared to the period of oscillations. At the same time the adiabatic region will be transformed to an isothermal one and the wave number will change from the adiabatic value to the isothermal one and this will account for the reflection. Because Newtonian cooling adds a new term to the linearized energy equation, damping modifies all linear relations among perturbation quantities. In particular, it decreases the wave amplitude and thereby the energy flux as well.

The nature of the dissipation introduced by Newtonian cooling is completely different from those of the viscosity and the thermal conduction. It exists only when there is a heat exchange between hotter and cooler regions in the atmosphere. Also the physical properties of the reflecting layer, created by the viscosity, will stay the same, i.e absorbing and reflecting, but the magnitude of the reflection coefficient will be changed from the adiabatic value to the isothermal one. Thus the attenuation in the amplitude of the waves and the changing factor in the magnitude of the reflection coefficient will vanish if the atmosphere is adiabatic or isothermal because these two regimes are dissipationless.

\section{STATEMENT OF THE PROBLEM}

Suppose an isothermal atmosphere, which is viscous and thermally conducting, occupies the upper half-space $z>0$. We will consider small oscillations about equilibrium which depend only on the time $\mathrm{t}$ and on the vertical coordinate $z$. Let $p, \rho, w$, and $T$ be the perturbations in the pressure, density, vertical velocity and temperature, and $P_{0}, \rho_{0}$, and $T_{0}$ are the equilibrium quantities. The equilibrium pressure and density,

$$
P_{0}(z) / P_{0}(0)=\rho_{0}(z) / \rho_{0}(0)=e^{-z / H},
$$

are determined by the gas law, $P_{0}=R T_{0} \rho_{0}$ and the hydrostatic equation, $P^{\prime}+g \rho_{0}=0$, where $\mathrm{R}$ is the gas constant, $\mathrm{g}$ is the gravitational acceleration, and $H=R T_{0} / g$ is the density scale height. The linearized equations of motion are:

$$
\begin{gathered}
\rho_{0} w_{t}+p_{z}+g \dot{\rho}=4 \mu w_{z z} / 3 \\
\rho_{t}+\left(\rho_{0} w\right)_{z}=0, \\
\rho_{0}\left(c_{V}\left(T_{t}+q T\right)+g H w_{z}\right)=\kappa T_{z z} \\
P=R\left(\rho_{0} T+T_{0} \rho\right) .
\end{gathered}
$$

Here $\mu$ is the dynamic viscosity coefficient, $\kappa$ is the thermal conductivity, $c_{V}$ is the specific heat at constant volume and $q$ is the Newtonian cooling which refers to the heat exchange and proportional to the temperature perturbation associated with the wave, all assumed to be constants. Equation (2.4) includes the heat flux term $c_{V} \rho_{0} q T$, which comes from the Stefan-Boltzman law. The subscripts $z$ and $t$ denote differentiations with respect to $z$ and $t$ respectively. We will consider solutions which are harmonic in time i.e $w(z, t)=W(z) \exp (-i \omega t)$ and $T(z, t)=T(z) \exp (-i \omega t)$. 
It is more convenient to rewrite the equations in dimensionless form; $z^{*}=z / H, \omega_{a}=c / 2 H$, $W^{*}=w / c, \omega^{*}=\omega / \omega_{a}, t^{*}=t \omega_{a}, \kappa^{*}=2 \kappa / c_{V} c H \rho_{0}(0), q^{*}=q / \omega_{a}, \mu^{*}=2 \mu /\left(3 \rho_{0}(0) c H\right), T^{*}=$ $T / 2 \gamma T_{0}$, where $c=\sqrt{\gamma R T_{0}}=\sqrt{\gamma g H}$ is the adiabatic sound speed, $\gamma$ is the ratio of the specific heats and $\omega_{a}$ is the adiabatic cutoff frequency The stars can be omitted, since all variables will be written in dimensionless form from now on.

One can eliminate $P$ and $\rho$ to obtain two equations for $W(z)$ and $T(z)$ by applying $\frac{\partial}{\partial t}$ to equation (2.1) and substituting equations $(2.2-2.4)$

$$
\begin{gathered}
\left(D^{2}-D+\gamma \omega^{2} / 4\right) W(z)+\gamma \eta e^{z} D^{2} W(z)+\imath \gamma(D-1) T(z)=0, \\
(\gamma-1) D W(z)=\gamma(i \omega-q) T(z)+\gamma \kappa e^{z} D^{2} T(z),
\end{gathered}
$$

where $D=d / d z$. Finally, $W(z)$ can be eliminated to obtain an equation for $T(z)$ only by differentiating equation (2.5) with respect to $\mathrm{z}$ and substituting for $\mathrm{D} \mathrm{W}(\mathrm{z})$ from equation (2.6):

$$
\begin{gathered}
{\left[\gamma \omega\left(D^{2}-D+\omega^{2} / 4\right)+i q\left(D^{2}-D+\gamma \omega^{2} / 4\right)-i \kappa e^{z} D^{2}\left(D^{2}+D+\gamma \omega^{2} / 4\right)\right.} \\
\left.-i \gamma(\omega+i q) \mu e^{z}\left(D^{2}+D\right)-\gamma \kappa \mu e^{2 z} D^{2}(D+1)(D+2)\right] T(z)=0 .
\end{gathered}
$$

In addition it is convenient to introduce the dimensionless parameter $\epsilon=\kappa / \mu, \epsilon \propto 1 / \operatorname{Pr}$ where $\operatorname{Pr}$ is the Prandtl number, which measures the relative strength of the effect of the viscosity with respect to that of the thermal conductivity. Consequently equation (2.7) becomes

$$
\begin{gathered}
{\left[\gamma \omega\left(D^{2}-D+\omega^{2} / 4\right)+i q\left(D^{2}-D+\gamma \omega^{2} / 4\right)-i \epsilon \mu e^{z} D^{2}\left(D^{2}+D+\gamma \omega^{2} / 4\right)\right.} \\
\left.-i \gamma(\omega+i q) \mu e^{z}\left(D^{2}+D\right)-\gamma \epsilon\left(\mu e^{z}\right)^{2} D^{2}(D+1)(D+2)\right] T(z)=0 .
\end{gathered}
$$

Equation (2.8) can be written in the following form

$$
\begin{gathered}
{\left[\left(D^{2}-D+Q \omega^{2} / 4\right)-Q i \mu e^{z}\left(D^{2}+D\right)\right.} \\
\left.\frac{\epsilon}{s} i \mu e^{z} D^{2}\left(D^{2}+D+\gamma \omega^{2} / 4\right)-\frac{\epsilon}{s} \gamma\left(\mu e^{z}\right)^{2} D^{2}(D+1)(D+2)\right] T(z)=0,
\end{gathered}
$$

where $Q=(\gamma \omega+i \gamma q) /(\gamma \omega+i q)$ and $s=\gamma \omega+i q$.

Boundary Conditions : To complete the formulation of the problem, certain conditions must be imposed to ensure a unique solution. Physically relevant solutions must satisfy the following boundary conditions as $z \rightarrow \infty$ :

$$
\mu \int_{0}^{\infty}\left|W_{z}\right|^{2} d z<\infty, \quad \kappa \int_{0}^{\infty}\left|T_{z}\right|^{2} d z<\infty .
$$

These are, respectively, the Dissipation Condition (DC) and the Entropy Condition (EC). The first one requires the finiteness of the rate of change of the energy dissipation in an infinite column of fluid of unit cross-section per period of oscillation. The second one requires the rate of change of the entropy in a column of fluid to be finite. Both of these conditions are necessary but not always sufficient.

Boundary conditions are also, required at $z=0$ and we shall adopt the lower boundary condition (LBC ): In a fixed interval $0<z<z_{0}$, the solution of the differential equation(2.9) should approach 
some solution of the limiting differential equation, $\left(D^{2}-D+Q \omega^{2} / 4\right) T(z)=0$. Considering the lower boundary condition is simpler than prescribing $T(z)$ and $W(z)$ at $z=0$, which necessitates the computation of the boundary layer near $z=0$. To first order the boundary layer has no effect on the reflection process, which takes place at high altitude. It will be seen that these boundary conditions determine a unique solution to the boundary value problem to within a multiplying constant.

We are interested in the problem when the viscosity is small but its effect is large compared to that of the thermal conduction and Newtonian cooling may take any positive value, i.e for small $\epsilon$ and $\mathrm{q} \geq 0$. For small $\mu$ the solutions of the the differential equation (2.9) will behave in the following way. There will be an "invicid region ", $0<z<<z_{1}=-\log \mu$, in which the kinematic viscosity $\mu e^{z}$ and the thermal diffusivity $\kappa e^{z}$ are small. As a result of that the solutions of the differential equation (2.9), in the invicid and above the boundary layer, can be approximated by the solutions of the following differential equation

$$
\left[D^{2}-D+Q \omega^{2} / 4\right] T(z)=0 .
$$

For any $\mu \neq 0$, no matter how small, there will be a "viscous region" in which the kinematic viscosity $\mu e^{z}$ is large while the thermal diffusivity is still small. Consequently the viscous terms will dominate for large $\mathrm{z}$ because of the exponential decrease of the density with altitude as $z \rightarrow \infty$ and the solutions of the differential equation (2.9), in the region $z_{1}<z<\infty$, can be approximated by the solutions of the differential equation

$$
\left[D^{2}(D+1)(D+2] T(z)=0 .\right.
$$

The "invicid" and the "viscous" regions are connected by a transition region, in the vicinity of $z_{1}$, in which the kinematic viscosity changes from small to large values and in it the reflection and the wave modification take place. As a result of that the solution of the differential equation (2.9), for small $\epsilon$, can be approximated by the solutions of the following differential equation

$$
\left[\left(D^{2}-D+Q \omega^{2} / 4\right)-Q\left(i \mu e^{z}\right)\left(D^{2}+D\right)-\frac{\epsilon}{s} \gamma\left(\mu e^{z}\right)^{2}\left(D^{2}(D+1)(D+2)\right] T(z)=0 .\right.
$$

It is clear that when $\mathrm{q} \rightarrow \infty$ the parameter $\mathrm{s} \rightarrow i \infty$ and $Q \rightarrow \gamma$. Consequently the last two terms of the differential equation (2.9) will vanish. Thus, in the limiting case, we expect the solution of the boundary value problem to be governed by the solutions of the following differential equation.

$$
\left[\left(D^{2}-D+Q \omega^{2} / 4\right)-Q\left(i \mu e^{z}\right)\left(D^{2}+D\right)\right] T(z)=0 .
$$

Our main problems are the connection of the solutions of the boundary value problem across the transition region, the investigation of the behaviour of the solution for large Prandtl number and the study of the influence of Newtonian cooling on the propagation of the waves when $q \geq 0$. The first problem will be considered in section (3) and the second one will be studied in section (4). The problem of the effect of Newtonian cooling on vertically propagating acoustic gravity waves in viscous and thermally conducting isothermal atmosphere for small Prandtl number and q may take any positive value will be considered in the second part of this series. 


\section{SOLUTION OF THE PROBLEM}

In this section we will investigate the problem of the connection of the solutions across the transition region. To do that it is convenient to introduce a new dimensionless variable

$$
\chi=\exp (-z) / \imath \mu=\exp (-z-\log \mu+3 \pi \imath / 2)
$$

which transforms the differential equation (2.9) into:

$$
\begin{gathered}
{\left[\chi^{2}\left(\Phi^{2}+\Phi+Q \omega^{2} / 4\right)-Q \chi\left(\Phi^{2}-\Phi\right)\right.} \\
\left.-\frac{\epsilon}{s}\left(\chi\left(\Phi^{2}-\Phi+\gamma \omega^{2} / 4\right)+\gamma(\Phi-1)(\Phi-2)\right) \Phi^{2}\right] T(\chi)=0
\end{gathered}
$$

where $\Phi=\chi d / d \chi$ and $\arg \chi=3 \pi / 2$. For fixed $\mu>0$, the point $\chi=0$ corresponds to $z=\infty$, the point $\chi_{0}=1 / i \mu$ to $z=0$ and the segment (with arg $3 \pi / 2$ ) connecting these points in the complex $-\chi$ plane to $z>0$. It is evident that the differential equation (3.2) leads to a singular perturbation problem. We will solve it by matching "inner" and "outer " approximations of the solutions in an overlapping domain. The differential equation for the outer approximation is obtained by letting $T(\chi)=U(\chi)+O(\epsilon)$ and setting $\epsilon=0$ in the differential equation ( 3.2 ), the resulting equation is

$$
\left[\chi\left(\Phi^{2}+\Phi+Q \omega^{2} / 4\right)-Q\left(\Phi^{2}-\Phi\right)\right] U(\chi)=0 .
$$

where $U(\chi)$ denote the outer approximation. Let $\chi=Q \xi$ and substitute for $\Phi$ then the differential equation (3.3) will be written in the following form

$$
\left[\xi(1-\xi) D^{2}-2 \xi D-Q \omega^{2} / 4\right] U(\xi)=0
$$

where $D=d / d \xi$. This differential equation is a special case of the hypergeometric equation with $\mathrm{c}=0, \mathrm{a}+\mathrm{b}=1$, and $\mathrm{ab}=Q \omega^{2} / 4$. Also it has three regular singular points at $\xi=0, \xi=1$ and $\xi=\infty$. The intermediate singular point $\xi=1$ corresponds to the transition layer. For $|\xi|<1$ its solution is a linear combination of

$$
U_{1}(\xi)=\xi F(a+1, b+1,2, \xi), \quad U_{2}(\xi)=U_{1}(\xi) \log \xi+(1 / a b)+\Sigma_{n=0}^{\infty} A_{n} \xi^{n+1},
$$

where $\mathrm{F}$ is the hypergeometric function, $\quad a=1 / 2+\sqrt{1-Q \omega^{2}}$ and $b=1 / 2-\sqrt{1-Q \omega^{2}}$. For $|\xi|>1$ it is convenient to choose

$$
U_{a}(\xi)=(-\xi)^{-a} F\left(a, a+1,2 a, \xi^{-1}\right) \quad U_{b}(\xi)=(-\xi)^{-b} F\left(b, b+1,2 b, \xi^{-1}\right) .
$$

The analytic continuations of $U_{a}(\xi)$ and $U_{b}(\xi)$ for small $\xi$ can be written in the following form (Abramowitz and Stegun, 1964, 15.3.14)

$$
\begin{gathered}
U_{a}=f_{a}\left[A(\xi)-\Sigma_{0}^{\infty} A_{n}(a) \xi^{n+1}\right], \quad U_{b}(\xi)=f_{b}\left[A(\xi)-\Sigma_{0}^{\infty} A_{n}(b) \xi^{n+1}\right] \\
A(\xi)=(1 / a b)-\log (-\xi) U_{1}(\xi), \quad f_{a}=b \Gamma(2 a) / \Gamma^{2}(a), \quad f_{b}=a \Gamma(2 b) / \Gamma^{2}(b) .
\end{gathered}
$$


The differential equation for the inner approximation can be obtained by introducing the stretching variable $\chi=\epsilon \eta$, letting $T^{\prime}(\chi)=\epsilon I(\eta)+O\left(\epsilon^{2}\right)$ and retaining the lowest terms in the differential equation (3.2). We obtain the following differential equation

$$
\left[\eta^{2} D^{2}-\eta D-s \eta\right] V(\eta)=0, \quad V(\eta)=\eta^{2} D^{2} I(\eta) .
$$

The differential equation for $V(\eta)$ can be transformed into the Bessel equation and it has two linearly independent solutions of the form

$$
V_{1}(\eta)=\eta J_{2}(2 \imath \sqrt{s \eta}) \quad V_{2}(\eta)=\eta Y_{2}(2 \imath \sqrt{s \eta}),
$$

where $J_{2}$ and $Y_{2}$ are Bessel functions of the first and second kind of order two. From the behaviour of $J_{2}$ and $Y_{2}$ for small argument, one can obtain the behaviour of the corresponding solutions of $I(\eta)$, (Abramowitz and Stegun, 1964, 9.1.9)

$$
I_{1}(\eta) \sim \eta^{2} \sim \exp (-2 z), \quad I_{2}(\eta) \sim \log \eta \sim z
$$

It is clear that $I_{1}$ satisfies the boundary conditions $(2.10)$ while $I_{2}$ does not. The behaviour of the solution for large argument $\eta$ may be obtained from the asymptotic form of the Hankel functions $H_{2}^{(1)}$ and $H_{2}^{(2)}$. Reintroducing the independent dimensionless variable $z$, via (3.1), we have approximately (Abramowitz and Stegun, 1964, 9.2.3, 9.2.4)

$$
V_{1}(z) \sim \exp [-(1+r) z+i r z], \quad V_{2} \sim \exp [(1-r) z-i r z] .
$$

where $r \approx \sqrt{|s| / 4 \kappa}$ ( when $\mathrm{q}=0 \mathrm{r} \approx \sqrt{\omega /(2 \kappa)})$. The first of these solutions corresponds to the boundary layer and it is significant only near $z=0$. It has no effect on the reflection process which takes place at high altitude and it is clear that Newtonian cooling reduces the width of the boundary layer. In fact, for fixed $z$ the boundary layer will tend to zero when $q \rightarrow \infty$. The second solution increases exponentially with $\mathrm{z}$ and cannot be matched with any solution of the differential equation(3.4). The remaining solutions of differential equation (3.9) are the solutions of the differential equation $\eta^{2} D^{2} I=0$. They are $C_{1}$ and $\eta$ i.e $C_{1}$ and $e^{-z} / i \kappa$. No one of these solutions satisfies the boundary conditions (2.10), but the linear combination

$$
I(\eta)=C_{1}+C_{2} \eta=1+s \eta
$$

does if $C_{2}=s C_{1}$. This can easily be shown from the dissipation condition and the differential equation (2.6). For simplicity we set $C_{1}=1$.

Finally the solution to the boundary value problem (equation 2.9) can be obtained by matching the solutions of the differential equation (3.9) as $\eta \rightarrow \infty$ which satisfy the boundary conditions (2.10) with the solutions of the differential equation $(3.4)$ as $\chi \rightarrow 0(\xi \rightarrow 0)$. The term $U_{1}(\xi) \log (-\xi)$ is matched in the second step of the matching procedure. Consequently we have

$$
c_{a} U_{a}+c_{b} U_{b}(\chi) \sim\left(f_{a} c_{a}+c_{b} f_{b}\right) /(a b)+\left[f_{a} A_{0}(a) c_{a}+f_{b} A_{0}(b)\right] \chi / Q \approx \epsilon(1+s \chi / \epsilon)=\epsilon+s \chi .(3.14)
$$


In the limiting case as $\epsilon \rightarrow 0$ we obtain

$$
\begin{gathered}
c_{a}=-s Q /\left(m f_{a}\right), \quad c_{b}=s Q /\left(m f_{b}\right) \\
m=A_{0}(b)-A_{0}(a)=\psi(a+1)+\psi(a-1)-\psi(b+1)-\psi(b-1), \\
\psi(x)=\Gamma^{\prime}(x) / \Gamma(x) .
\end{gathered}
$$

As a conclusion of this section the solution of the boundary value problem (equation 2.9), in the region $0<z<z_{1}$, can be written in the following form

$$
T(\chi)=c_{a} U_{a}(\chi)+c_{b} U_{b}(\chi) \sim c_{a}(-\chi)^{-a}+c_{b}(-\chi)^{-b} .
$$

\section{THE BEHAVIOUR OF THE SOLUTION}

In this section will investigate the behaviour of the solution of the boundary value problem (equation 2.9) below and above the reflecting layer produced by the viscosity. In section three we have concluded that the solution can be written in the form of equation (3.17). Reintroducing the dimensionless variable $\mathrm{z}$ via (3.1) and substituting in equation (3.17) we have

$$
T(z) \sim c_{a}[\exp (a z)+R c \exp (b z)],
$$

where $a=1 / 2+\sqrt{1-Q \omega^{2}} / 2, b=1 / 2-\sqrt{1-Q \omega^{2}} / 2, \theta=\arg \left(b \Gamma^{2}(b) \Gamma(2 a)\right)$. and

$$
R c=\exp [i \pi(a-b) / 2+(b-a) \log (\mu Q)+2 i \theta]
$$

( Here and elsewhere $a, b, R c$ and $\theta$ denote constants which can assume different values in different expressions).

It is well known that radiative damping is a dissipative process. To investigate the nature of the dissipation and its effects on the behaviour of the solution for positive $q$, it is convenient to write the parameter $\mathrm{Q}$ in the following form

$$
Q=\gamma(\omega+i q) /(\gamma \omega+i q)=1+i(q / \omega)(\gamma-1) / \gamma-\ldots \ldots
$$

Thus for $\omega>\omega_{a}$ we have

$$
\sqrt{1-Q \omega^{2}} / 2=i \sqrt{4 k_{a}^{2}+i q \omega(\gamma-1) / \gamma+\ldots .}= \pm\left(-a(q)+i k_{a}\right),
$$

where $\mathrm{a}(\mathrm{q})$ denote the attenuation factor. Consequently the solution has the following form

$$
T(z) \sim c_{a}\left[\exp \left[\left(1 / 2-a(q)+i k_{a}\right) z\right]+R C_{q} \exp \left[\left(1 / 2+a(q)-i k_{a}\right) z\right]\right]
$$

where the reflection coefficient $R C_{q}$ defined by

$$
\begin{gathered}
R C_{q}=\exp \left[N+i M-\pi k_{a}\right] \\
N=2\left[a(q) \log |\mu Q|+k_{a} \theta_{q}\right], \quad M=2\left[\theta+a(q) \theta_{q}-k_{a} \log |\mu Q|-\pi a(q) / 2\right],
\end{gathered}
$$


and $\theta_{q}=\arg (Q)$, its maximum is $(\gamma-1) /(2 \sqrt{\gamma})$ and attains when $\omega / q=\sqrt{\gamma}$.

The first term on the right of equation (4.4) represents an upward propagating wave, its amplitude decaying with altitude like $\exp [-\mathrm{a}(\mathrm{q}) \mathrm{z}]$, while the second term is a downward travelling wave decaying at the same rate. Equation (4.4) describes the behaviour of solution, the reflection process and the attenuation in the amplitude of the wave for all values of $q$ below the reflecting layer. Above the reflecting layer the solution will decay with altitude to a constant value. The reflection takes place in the vicinity of $z_{1}=-\log |\mu Q|$. The value of the parameter $\mathrm{N}$ is small and negative. Its existence depends on the heat exchange between any two regions with different temperature in the atmosphere. It will vanish if the atmosphere is either adiabatic or isothermal. The factor $\exp [\mathrm{N}]$ in equation (4.5) represent the attenuation factor in the magnitude of the reflection coefficient.

To put the conclusions of this paper in a more physical setting, it is important to note the following two limiting cases

CASE A : when $q=0$ the parameter $Q$ reduces to 1 and for frequencies greater than the adiabatic cutoff frequency $\omega_{a}$ we have $a=1 / 2+i k_{a}, b=1 / 2-i k_{a}$, where $k_{a}=\sqrt{\omega^{2}-1} / 2$ is the adiabatic wave number, and equation (4.4) will be written in the following form

$$
T(z) \sim c_{a}\left[\exp \left[\left(1 / 2+i k_{a}\right) z\right]+R C_{a} \exp \left[\left(1 / 2-i k_{a}\right) z\right]\right],
$$

where the reflection coefficient $R C_{a}$ is defined by

$$
R C_{a}=\exp \left[2 i\left(\theta_{a}-k_{a} \log (\mu)\right)-\pi k_{a}\right], \quad\left|R C_{a}\right|=\exp \left[-\pi k_{a}\right]
$$

Equation (4.7) describes the behaviour of the solution in the adiabatic region. It states that the solution of the boundary value problem below the reflecting layer, created by the viscosity, is a linear combination of an upward and a downward propagating wave with an adiabatic wavelength $\lambda_{a}=2 \pi / k_{a}$.

CASE B: when $q \rightarrow \infty$ the parameter $\mathrm{Q} \rightarrow \gamma$ and for frequencies greater than the isothermal cutoff frequency $\omega_{i}=1 / \sqrt{\gamma}$ we obtain $a=1 / 2+i k_{i}$, and $b=1 / 2-i k_{i}$ where $k_{i}=\sqrt{\gamma \omega^{2}-1} / 2$ is the isothermal wave number. Consequently the solution will be written like

$$
T(z) \sim c_{a}\left[\exp \left[\left(1 / 2+i k_{\mathbf{\imath}}\right) z\right]+R C_{\mathbf{\imath}} \exp \left[\left(1 / 2-i k_{\mathbf{\imath}}\right) z\right]\right],
$$

and the reflection coefficient $R C_{\mathrm{z}}$ can be written as

$$
R C_{\imath}=\exp \left[2 i\left(\theta_{\mathbf{\imath}}-k_{\mathbf{\imath}} \log (\gamma \mu)\right)-\pi k_{\mathbf{\imath}}\right], \quad\left|R C_{i}\right|=\exp \left[-\pi k_{\mathbf{\imath}}\right] .
$$

From equations (4.5), (4.8), and (4.10) we have

$$
\exp \left[-\pi k_{i}\right] \leq\left|R C_{q}\right| \leq \exp \left[-\pi k_{a}\right]
$$

Equation (4.9) has the same interpretation for the behaviour of the solution below the reflecting layer as that of equation (4.7) except that the wavelength is changed to the isothermal value $\lambda_{2}=2 \pi / k_{2}$, which indicates the transformation of the adiabatic region to the isothermal form. 
We observe from these two cases that when $q=0$ or $q \rightarrow \infty$ there is no attenuation in the amplitude of the wave and for fixed $\omega$, both reflection coefficients are periodic functions of $\log (\mu)$

$$
\log (\mu)=\left(\theta_{a} \pm 2 n \pi\right) / k_{a} \quad \log (\gamma \mu)=\left(\theta_{1} \pm 2 n \pi\right) / k_{\mathbf{i}}
$$

In addition to that as $q \rightarrow \infty$ the wave number and the cutoff frequency change from the adiabatic values to the isothermal ones. Thus Newtonian cooling influences only the adiabatic region and transforms it to the isothermal form.

\section{CONCLUSIONS}

Before ending this paper we would like to indicate the main results

(I) For large Prandtl number the viscosity creates an absorbing and reflecting layer. Below it the oscillatory motion is adiabatic and above it the motion will decay exponentially with the altitude to zero before it is influenced by the effects of the thermal conductivity. Also as $q \rightarrow \infty$ the parameter $s \rightarrow i \infty$ and the last two terms of equation (2.9) will vanish. Consequently, for large Prandtl number, the effects of the thermal conductivity can be excluded.

(II) The magnitude of the reflection coefficient $\left|R C_{a}\right|\left(\left|R C_{\mathrm{a}}\right|\right)$ tends to 1 (its maximum value ) as $\lambda_{a} \rightarrow 2 \pi / k_{a} \rightarrow \infty\left(\lambda_{i}=2 \pi / k_{2} \rightarrow \infty\right)$, and to 0 as $\lambda_{a} \rightarrow 0\left(\lambda_{2} \rightarrow 0\right)$. The reflection becomes quite small when $\lambda_{a}\left(\lambda_{2}\right)$ equal to $\mathrm{H}$. Although $\left|R C_{a}\right|\left(\left|R C_{2}\right|\right)$ tends to a limit as $\mu \rightarrow 0$, the solution itself does not approach a limiting value at any fixed point $z$ of the altitude because of the oscillation of the phase between the two waves. This can be explained by the fact that the region from which most of the reflection takes place depends on the kinematic viscosity and shifts upward as $\mu \rightarrow 0$. Of course the same conclusions applied for each fixed value of $q$.

(III) It is clear from equation (2.6) that, with $\kappa=0$ and $q>>\omega$, we have $T \propto\left[W_{z} / q\right]$. Thus as $\mathrm{q} \rightarrow \infty$ we have $\mathrm{T} \rightarrow 0$. As a result of that when the heat exchange, due to radiation, is intense enough the temperature perturbation associated with the wave will be eliminated.

(IV) The nature of the dissipation introduced by Newtonian cooling is of a different type from those of the thermal conduction and vicosity because the existence of it depends on the heat exchange between hotter and cooler regions in the atmosphere.

(V) The nature of the reflecting layer created by the viscosity will not change as $q \rightarrow \infty$, but the magnitude of the reflection coefficient will be reduced from the adiabatic value to the isothermal one. The reduction in the magnitude of the reflection coefficient will continue until the atmosphere is transformed from the adiabatic regime to the isothermal one.

ACKNOWLEDGEMENT : I would like to express my sincere thanks to Professor Michael Yanowitch for his support and invaluable criticism during the preparation of this work. 


\section{REFERENCES}

1. ABRAMOWITZ, M.,\& STEGUN, I., Handbook of Mathematical Functions, National Bureau of Standards, Washington, D.C. 1964.

2. ALKAHBY, H.Y., \& YANOWITCH, M., The effects of Newtonian cooling on the reflection of vertically propagating acoustic waves in an isothermal atmosphere, Wave Motion 11 (1989), 419-426.

3. ALKAHBY, H.Y., \& YANOWITCH, M, Reflection of vertically propagating waves in a thermally conducting isothermal atmosphere with a horizontal magnetic field, Geophys. Astroph. Fluid Dynam. 56 (1991), 227-235.

4. ALKAHBY, H.Y., On the coronal heating mechanism by the resonance absorption of Alfven waves, to appear in Internat. J. Math. \& Math. Sci.

5. ALKAHBY, H.Y., Reflection and dissipation of hydromagnetic waves in a viscous and thermally conducting isothermal atmosphere, to appear in Geophys. Astroph. Fluid Dynam.

6. BUSSE, F.H., Thermal instabilities in rapidly rotating systems, J. Fluid. Mech. $\underline{44}$ (1970), 441-460.

7. BUSSE, F.H, A model of the geodynamo, Geophys. J.R. Astr. Soc. $\underline{42}$ (1975), 437-459.

8. CALLY, P.S., Magnetohydrodynamic critical levels and radiative damping, Astr. Astroph. 136 (1984), 121-126.

9. CAMPOS, L.M.C., On viscous and resistive dissipation of hydrodynamic and hydromagnetic waves in atmospheres, J. Mec. Theror. Appl. 2(1983b), 861-891.

10. CAMPOS, L.M.C On magnetoacoustic- gravity waves propagating or standing vertically in an atmosphere, J. Phys. A 16 (1983a), 417-437.

11. DEBNATH, L.,\& BASU, U., Capillary-gravity waves against a vertical cliff, Ind. $\underline{\text { J. Math }} \underline{26}$ (1984), 49.

12. ELTAYEB, I.A.\& ROBERTS, P.H., On the hydromagnetics of rotating fluids, J. Astrophys. $\underline{162}(1970)$ 699-701.

13. HOLLWEG, J.V., Resonance of coronal loop, J. Astrophys. 277 (1984) 392-403.

14. IONSON, J.A., Resonant electrodynamic heating of stellar loops, J. Astrophys. 254 (1982) 318-334.

15. LYONS, P., \& YANOWITCH, M., Vertical oscillations in a viscous and thermally conducting isothermal atmosphere, J. Fluid Mech. $\underline{66}$, (1974), 273-288. 
16. LINDZEN, R.S. Vertically propagating waves in an atmosphere with Newtonian cooling inversely proportional to density, Canad. J. Phys. 46, (1968), 1835-1840.

17. LINDZEN, R.S., Internal gravity waves in atmospheres with realistic dissipation and tempe ature, Part I, Geophys Fluid Dynamics 1 (1970), 303-355.

18. MIHALAS, B. W. \& TOOMRE, J., Internal gravity waves in the solar atmosphere ( part I. Adiabatic waves in the Chromosphere), AP. J. 249 (1981), 249-349.

19. MIHALAS, B. W., \& TOOMRE, J., Internal gravity waves in the solar atmosphere ( part II. Effects of radiative damping), AP. J. 263 (1982) 263-286.

20. MOFFATT, H.R., Magnetic field in electrically conducting fluids, Cambridge University Press, (1979).

21. PARKER, E.N., Cosmical Magnetic Fields, Their origin and their activity, Clarendon Press. Oxford 1979.

22. PRIEST, E.R., Solar Magnetohydrodynamics, D. Reidel Pub. Co. 1984.

23. ROBERTS, B.H., On the thermal instability of a rotating fluid sphere containing heat sources, Phil. Trans. R. Soc. Lond. A263, (1968) 93-117.

24. SOWARD, A.M., Non-linear marginal convection in a rotating magnetic system, Geophys. Astrophys. Fluid Dynam. 35 (1986), 329-371.

25. SUMMERS, D., Gravity modified sound waves in a conducting stratified atmosphere, Q. J. Mech. Appl. Math. 29 (1976), 117-126.

26. WEBB, A.R. \& ROBERTS,B.H., Vertical motion in an intense magnetic flux lube part IV,

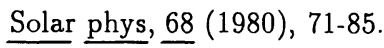

27. YANOWITCH, M., Effect of viscosity on vertical oscillations of an isothermal atmosphere, Canad. J. Phys. $\underline{45}$ (1967), 2003-2008.

28. YANOWITCH, M., Effect of viscosity on gravity waves and the upper boundary conditions, J. Fluid.Mech. 29 (1967), 209-231.

29. YANOWITCH, M., Vertically propagating hydromagnetic waves in an isothermal atmosphere with a horizontal magnetic field, Wave Motion 1 , (1979), 123-125.

30. ZHUGZHDA, YU.D.,\& DZHALILOV, N.S., Magneto-acoustic gravity waves in a horizontal magnetic field, Geophys. Fluid Dynamics. 35, (1986) 131-156. 


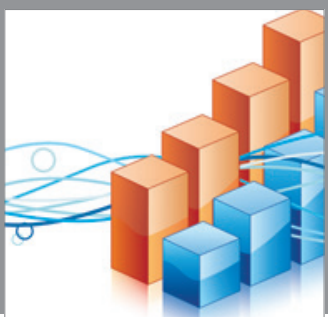

Advances in

Operations Research

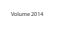

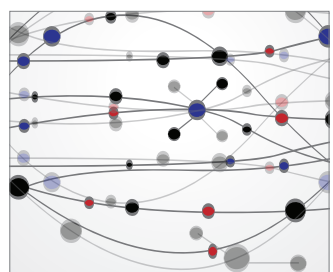

\section{The Scientific} World Journal
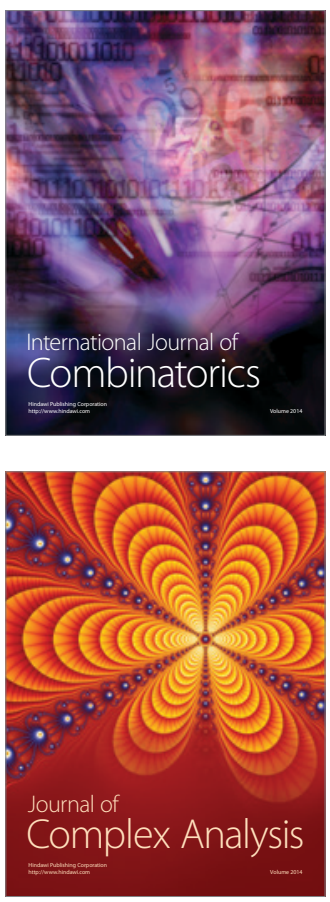

International Journal of

Mathematics and

Mathematical

Sciences
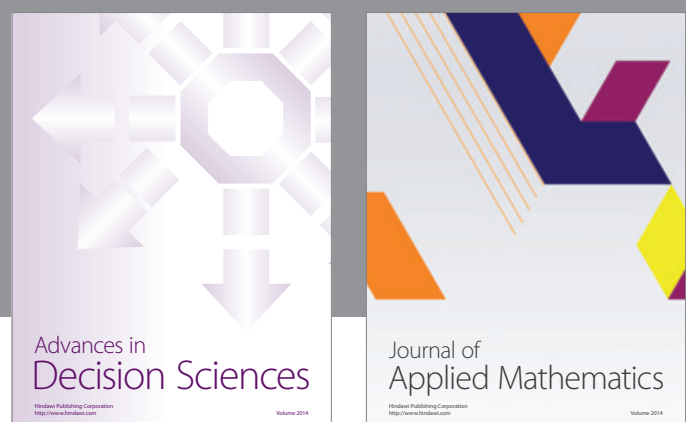

Journal of

Applied Mathematics
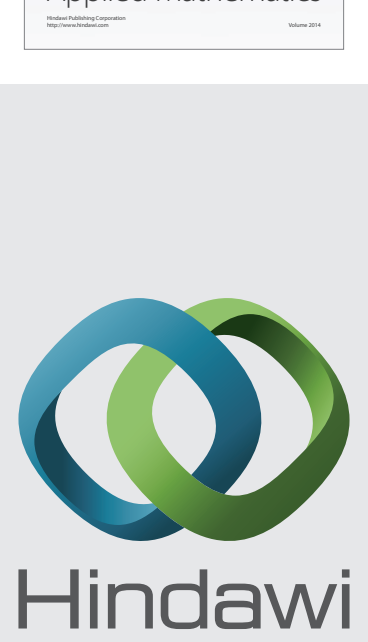

Submit your manuscripts at http://www.hindawi.com
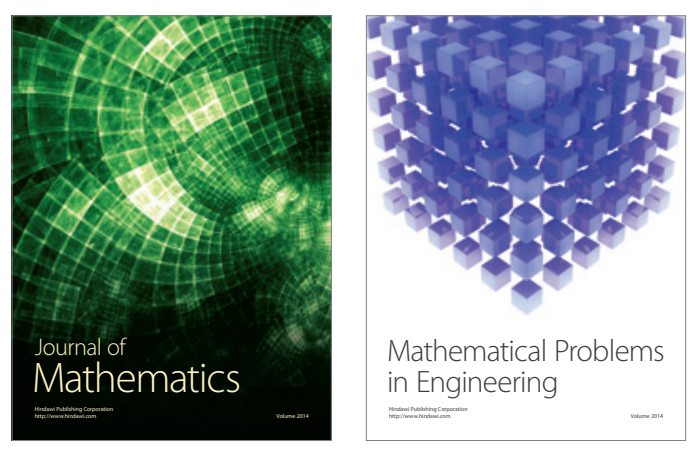

Mathematical Problems in Engineering
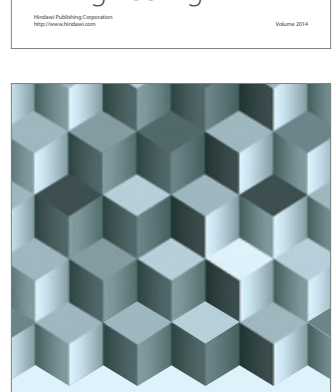

Journal of

Function Spaces
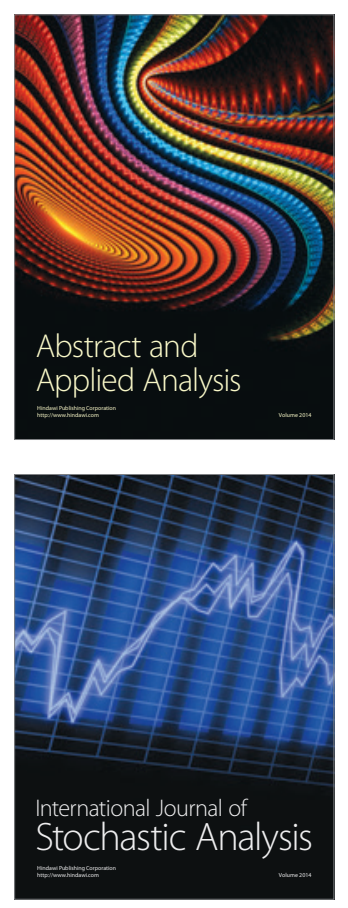

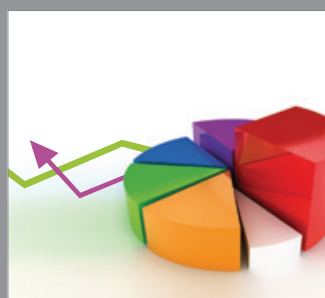

ournal of

Probability and Statistics

Promensencen
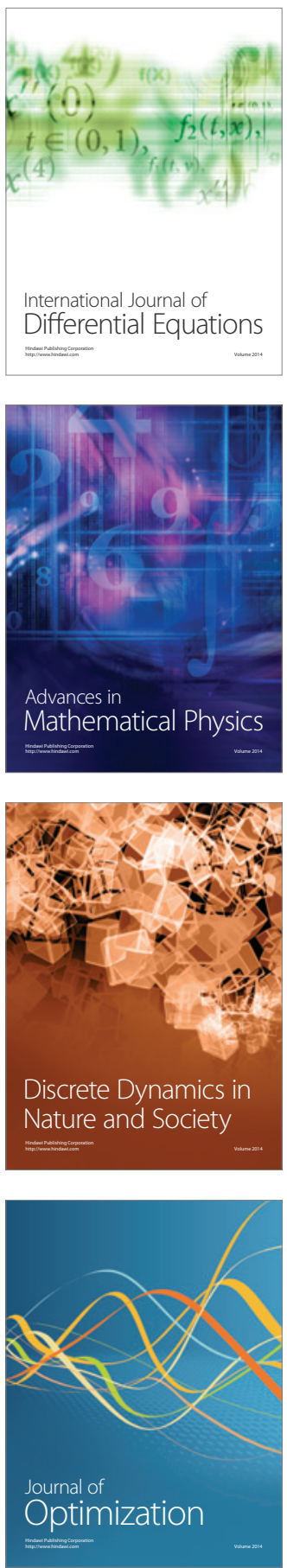